\title{
MAXIMIZAÇÃO DO PROCESSO PRODUTIVO EM UMA FÁBRICA TÊXTIL COM APLICAÇÃO DO SOFTWARE LINDO
}

\author{
Hériston Byron (UNIFAVIP-WYDEN) heristonbyron@hotmail.com \\ Jeconias dos Santos (UNIFAVIP-WYDEN) jcnsconias84@live.com \\ João Victor (UNIFAVIP-WYDEN) joaovictor8428@hotmail.com \\ Jean Turet (UNIFAVIP-WYDEN) jeanturet@gmail.com
}

Resumo: Na pesquisa operacional há um conjunto de ferramentas para análise e solução de problemas uma delas é a programação linear, é por meio dela que consiste em métodos para resolver problemas de otimização contendo restrições. A PL tornou-se uma ferramenta muito utilizada em empresa devido ao fato de reduzir custos ou aumentar os lucros, além da facilidade nas tomadas de decisões. Nesse artigo a Programação Linear foi utilizada para maximizar o lucro de uma empresa do setor têxil, a qual possui quatro produtos principais para compor seus ganhos (Calça masculina, Calça feminina, Bermuda masculina, Short feminino). Essa organização está localizada em Caruaru-PE e para elaborar o modelo matemático foi utilizado o LINDO®, junto com a programação inteira.

Palavras chave: Pesquisa Operacional, Programação Linear, Programação Inteira, Software LINDO®.

Maximizing the production process in a textile factory with LINDO® software application

Abstract: In operational research there is a set of tools for analysis and problem solving. One of them is linear programming, it is through it that it consists of methods to solve optimization problems containing constraints. PL has become a widely used business tool because it can reduce costs or increase profits, as well as easy decision making. In this article, Linear Programming was used to maximize a company's profit in the textile industry, which has four main products to make up its earnings (Men's Pants, Women's Pants, Men's Bermuda Shorts, Women's Shorts). This organization is located in CaruaruPE and to elaborate the mathematical model was used the LINDO ${ }^{\text {, }}$, along with the integer programming.

Keywords: Operational Research, Linear Programming, Integer Programming, LINDO® Software. 


\section{Introdução}

A globalização tornou o mercado mais exigente e competitivo, essa competitividade tem exigido das empresas a busca contínua em aprimorar a qualidade em todos os processos e atividades que executam, a fim de alcançar à aceitação dos seus produtos ou serviços, visando sempre a permanência no mercado em que atuam. De fato, existem alguns aspectos que influenciam fortemente na obtenção dos objetivos das empresas, são aqueles que dizem respeito à análise dos custos e suas influências nas decisões que impactam a formação do preço de venda.

O preço de venda deixou de ser objeto de decisões empíricas, mas, sobretudo, objeto de estudo, o que torna necessário conhecer todos os elementos que o compõem, sejam internos ou externos. Por isso a necessidade de conhecer não somente a metodologia de formação de preços de venda, mas também definir qual sistema de rateio de custos mais adequado a realidade.

Neste cenário está o Polo de confecção do agreste de Pernambuco o segundo maior do país e um dos maiores impulsionadores da economia no interior do Estado, se mostrou resiliente nas últimas décadas. Apesar da crise econômica que afetou o país nos últimos anos e a crise hídrica na região, o Polo, segundo o Diário de Pernambuco em 2017 movimentou $\mathrm{R} \$ 28,3$ bilhões, $17 \%$ do total brasileiro. Mas, grande parte da produção da região se dá na informalidade, como demonstra a Folha de São de Paulo em texto de 2019, deixando este grande contingente aquém de técnicas de otimiizaçao e auxilio na tomada de decisões.

Para isto serve a Pesquisa Operacional, com um conjunto de técnicas que através de modelos matemáticos, probabilísticos e algoritmos computacionais, consiste em um método cientifico para a tomada de decisao (Belfiore e Fávero, 2013).

Foi aplicado o estudo de pesquisa operacional inteira em uma empresa têxtil localizada em Caruaru-PE com intuito de encontrar o mix de produção que melhor proporciona a alocação mais eficiente dos recursos produtivos no processo de manufatura, visando a otimização da utilização dos recursos e do desempenho do sistema produtivo, gerando assim mais lucro a empresa. 


\section{Metodologia}

A metodologia escolhida foi um estudo de caso através de informações disponibilizadas pela empresa têxtil de pequeno porte, localizada na cidade de Caruaru-PE. O embasamento teórico para fundamentação desta pesquisa será através de publicação científica, ou seja, revisão da bibliografia, já a análise, foi descrita através de uma modelagem matemática, contendo as variáveis do problema, função objetivo e restrições. Utilizou-se a ferramenta software LINDO® para a resolução do modelo. Analisaram-se os resultados obtidos e eles foram organizados em formas de tabelas com o auxílio do programa Microsoft Office Excel®; Godoy (1995) caracteriza o método do Estudo de Caso como uma estratégia de produção preferida quando os pesquisadores procuram responder questões 'como' e 'por quê' certos fenômenos ocorrem, quando há pouca possibilidade de controle sobre os eventos estudados e quando o foco de interesse é sobre fenômenos atuais, que poderão ser analisados dentro de algum contexto da vida real.

\section{Fundamentação teórica}

\section{1 Pesquisa operacional}

A Pesquisa Operacional, surgiu na Segunda Guerra Mundial a partir da necessidade militar de solucionar problemas relacionados a ordem estratégica e tática como a distribuição de radares, dimensionamento de frotas, alimentação das tropas, manutenção e inspeção de aviões dentre outras atividades, convocando um grupo de cientistas para resolução destes problemas teve-se a primeira atividade formal desse campo (Belfiore e Fávero, 2013).

No pós-guerra a Pesquisa Operacional se desenvolveu e passou a ser aplicada à diversos problemas nos setores públicos e privados, no campo industrial e financeiro, como na mineração, construção civil, militar, têxtil, metalúrgica, transporte e bancário (Arenales et al., 2015).

Atualmente a Pesquisa Operacional reúne inúmeras ferramentas que se subdivide em modelos como: determinísticos, estocásticos e outras tecnicas. Segundo Santos et al. (2016) a Pesquisa Operacional atua em cinco grandes áreas que são, Avaliação Operacional de Sistemas, Apoio a Tomada de Decisões, Estruturação de Problemas, Otimização de Processos Produtivos e Simulação de Processos. 
Para Silva et. al (2010), Pesquisa Operacional, em linhas gerais, consiste na descrição de um sistema organizado com o auxílio de um modelo, e através da experimentação com o modelo, na descoberta da melhor maneira de operar o sistema. Segundo Moreira (2010), Cabe a PO determinar a condução e coordenação de algumas operações nas empresa.

Já para Hillier e Liberman (2013), a Pesquisa Operacional é uma ciência baseada em modelos que visam programar e coordenar as operações de uma organização. Ela trabalha analisando as variáveis e as restrições de um determinado problema real, com a finalidade de encontrar uma solução ótima.

\section{2 Programação Linear}

A Programação Linear em linhas gerais trata do problema de alocação ótima de recursos escassos para determinadas atividades que disputam os mesmos, por ótimo entenda-se, melhor das alternativas viáveis (Colin, 2018).

A Programação Linear utiliza modelos matemáticos com funções estritamente lineares para descrever o problema em questão. Embora a alocação de recursos seja sua principal aplicação ela não se restringe a isso, podendo ser utilizada em qualquer problema cujo modelo matemático se encaixe em seu formato genérico (Hillier e Liberman, 2013).

\section{2 Programação Linear Inteira}

A programação inteira pode ser entendida como um caso específico da programação linear, em que todas ou alguma(s) das suas variáveis são discretas (têm de assumir valores inteiros).

Segundo Lachtermacher (2016), programação inteira é a programação matemática em que uma ou mais variáveis de decisão são representadas apenas por valores inteiros, ou seja, é uma forma de programação linear onde as variáveis podem apenas apresentar números inteiros onde tem sido muito utilizado na resolução de problemas de investimento dentre outros. Um problema de programação linear inteira total pode ser descrito como: 
Otimizar $\mathrm{Z}=\mathrm{f}(\mathrm{x} 1, \mathrm{x} 2, \ldots, \mathrm{xn})$

$\left.\begin{array}{rr}\text { Sujeito a: } & g 1(x 1, x 2, \ldots, x n) \\ & g 2(x 1, x 2, \ldots, x n) \\ & g m(x 1, x 2, \ldots, x n)\end{array}\right] \cdot\left[\begin{array}{ll}\leq & b 1 \\ = & b 2 \\ \geq & b m\end{array}\right.$

x1, x2, x3 ...,xn são inteiros

Onde:

$\mathrm{f}(\mathrm{x} 1 ; \mathrm{x} 2 ; ; \mathrm{x} 3 ; \ldots \mathrm{xn})=\mathrm{c} 1 \mathrm{x} 1+\mathrm{c} 2 \times 2 ;+\mathrm{c} 3 \times 3+\ldots+\mathrm{cn} \times \mathrm{n}$

N são variáveis;

M são restrições;

I é o índice da restrição $(i=1,2 \ldots \mathrm{m})$;

J é o índice da variável $(\mathrm{j}=1,2 \ldots \mathrm{n})$;

Ci é o coeficiente da variável xi da função-objetivo;

Aij é o coeficiente da variável xi da j-ésima restrição.

\section{3 Função objetivo}

Durante a formulação do problema, a combinação de variáveis a que se chega é colocada na forma de uma expressão matemática, que recebe o nome de função objetivo (MOREIRA, 2011). A função objetivo depende das variáveis de decisão que são incógnitas quantificáveis sejam elas máquinas, produtos ou grandezas de processo, ou seja, como o próprio nome diz indica o objetivo que se deseja atingir a partir das variáveis de decisão.

\section{4 Variáveis de decisão}

As variáveis de decisão são os valores a serem encontrados no modelo. Os parâmetros representam as variáveis controladas do sistema. De acordo com o objetivo exercido na modelagem as variáveis de decisão exemplificam produtos, máquinas etc. Essas variáveis serão valores que buscam sempre a melhor solução para o problema em questão. 


\section{5 Restrições do problema}

Estão ligadas às limitações físicas do sistema, o modelo deve incluir restrições que limitam as variáveis de decisão a seus valores possíveis (ou viáveis), sejam elas a quantidade de funcionários, tempo de máquina, quantidade matéria prima, capacidade produtiva, capacidade de armazenamento, dentre outros.

As restrições também estão divididas em LHS (left hand side) que é o lado esquerdo da restrição, onde apresenta a fórmula e em seguida o seu resultado e RHS (right hand side) que é o lado direito da restrição, com sua respectiva constante (AMOURY NETA et al., 2014).

\section{6 Software LINDO®}

Por ser considerada uma das melhores ferramentas para resolver problemas de otimização maior facilidade e velocidade na sua utilização, foi optado por utilizar com LINDO® (Linear Interactive Discrete Optimizer), cujo objetivo é maximizar o lucro e minimizar o custo em decisões envolvendo planejamento de produção, transporte, finanças, alocação de portfólio, orçamento de capital, programação, inventário, recurso alocação e muito mais, ou seja fazer pequenas modificações nos dados ou parâmetros, e obter um ótimo resultado para qualquer organização.

Como o objeto do estudo é a produção de peças Jeans, torna-se necessário entender o principal problema do mesmo. Um problema de produção seja ela qual for, refere-se a dois parâmetros principais, utilização de insumos, e o tempo de produção. Consistindo em otimizar a produção, buscaram-se menor quantidade possível de insumos, e menor tempo de produção.

\section{Estudo de caso}

Com o intuito de aplicar os conceitos trabalhados no decorrer do artigo, escolheu-se uma empresa têxtil de pequeno porte localizada na cidade de Caruaru-PE, na qual o objetivo principal da organização era estabelecer uma programação mensal de produção visando alcançar a maximização nesse determinado intervalo de tempo, que foi feito para quatro tipos de modelos, em que a empresa produz: Calças Jeans Masculina(X1) e Feminina(X2), Bermudas Jeans Masculina(X3) e Shorts Feminino(X4), que apresentam respectivamente os lucros por unidade: $\mathrm{R} \$ 59,00,49,00,27,90,22,90$. 
Para a produção das peças são necessários verificarmos as seis restrições que ocorrem nesse processo, onde três dessas restrições são recursos, como mostra a Tabela 1:

Tabela 1- Recursos Disponíveis

\begin{tabular}{cc}
\hline Recurso & Disponibilidade \\
\hline Jeans $\left(\mathrm{m}^{2}\right)$ & 10000 \\
Fios $(\mathrm{m})$ & 500000 \\
Mão de obra(min) & 24960 \\
Demanda (Calça masculina) & 350 \\
Demanda (Calça feminina) & 460 \\
Demanda (Bermuda masculina) & 100 \\
Demanda (Short feminino) & 50 \\
\hline
\end{tabular}

Fonte: Elaborado pelos autores

A utilização das quantidades dos recursos necessárias para confeccionar cada modelo de peças jeans está exposto na Tabela 2:

Tabela 2- Utilização dos recursos

\begin{tabular}{cccc}
\hline Produtos & Jeans $\left(\mathbf{m}^{\mathbf{2}}\right)$ & Fios $(\mathbf{m})$ & Mão de obra(min) \\
\hline Calça masculina & 1,6 & 160 & 32,2 \\
Calça feminina & 1,4 & 140 & 26,0 \\
Bermuda masculina & 1,3 & 120 & 23,0 \\
Short feminino & 1,1 & 110 & 20,5 \\
\hline
\end{tabular}

Fonte: Elaborado pelos autores

Com os valores unitários de cada peça, foi calculado o custo em reais de insumos por unidade de peça, como mostra na Tabela 3:

Tabela 3- Custos dos recursos

\begin{tabular}{cc}
\hline Recurso & Preço \\
\hline Jeans $\left(\mathrm{m}^{2}\right)$ & 10,00 \\
Fios(m) & 0,02 \\
Mão de obra(min) & 0,14 \\
\hline
\end{tabular}


Com base nesses dados foi feito a multiplicação desses custos que é informado na "Tabela 3" com a utilização dos recursos expostos na "Tabela 2", como mostra na Tabela 4, onde a soma é em $(\mathrm{R} \$)$.

Tabela 4- Custos por peça

\begin{tabular}{|c|c|c|c|c|}
\hline Produtos & $\operatorname{Jeans}\left(\mathbf{m}^{2}\right)$ & $\operatorname{Fios}(\mathbf{m})$ & $\begin{array}{c}\text { Mão de } \\
\text { obra(min) }\end{array}$ & Soma \\
\hline Calça masculina & $1,6 \times 10$ & $160 \times 0,02$ & $32,2 \times 014$ & 23,71 \\
\hline Calça feminina & $1,4 \times 10$ & $140 \times 0,02$ & $26,0 \times 0,14$ & 20,44 \\
\hline Bermuda masculina & $1,3 \times 10$ & $120 \times 0,02$ & $23,0 \times 0,14$ & 18,62 \\
\hline Short feminino & $1,1 \times 10$ & $110 \times 0,02$ & $20,50,14$ & 16,07 \\
\hline
\end{tabular}

Fonte: Elaborado pelos autores

Por fim será subtraído do preço unitário de cada peça, encontrando assim o ganho unitário por peça, como é exposto na Tabela 5.

Tabela 5- Ganhos reais

\begin{tabular}{cccc}
\hline \multicolumn{1}{c}{ Produtos } & Preço vendido & Gastos & Ganhos \\
\hline Calça masculina & $\mathrm{R} \$ 59,00$ & $\mathrm{R} \$ 23,71$ & $\mathrm{R} \$ 35,29$ \\
Calça feminina & $\mathrm{R} \$ 49,00$ & $\mathrm{R} \$ 20,44$ & $\mathrm{R} \$ 28,56$ \\
Bermuda masculina & $\mathrm{R} \$ 27,90$ & $\mathrm{R} \$ 18,62$ & $\mathrm{R} \$ 9,28$ \\
Short feminino & $\mathrm{R} \$ 22,90$ & $\mathrm{R} \$ 16,07$ & $\mathrm{R} \$ 6,83$ \\
\hline
\end{tabular}

Fonte: Elaborado pelos autores

\section{1 Modelagem}


Com base nesses dados, foi montado a seguinte modelagem matemática onde (Calça masculina $(\mathrm{CM})=\mathrm{x} 1$, Calça feminina $(\mathrm{CF})=\mathrm{x} 2$, Bermuda masculina $(\mathrm{BM})=\mathrm{x} 3$ e Short feminino $(\mathrm{SF})=\mathrm{x} 4)$, como mostra na Tabela 6 :

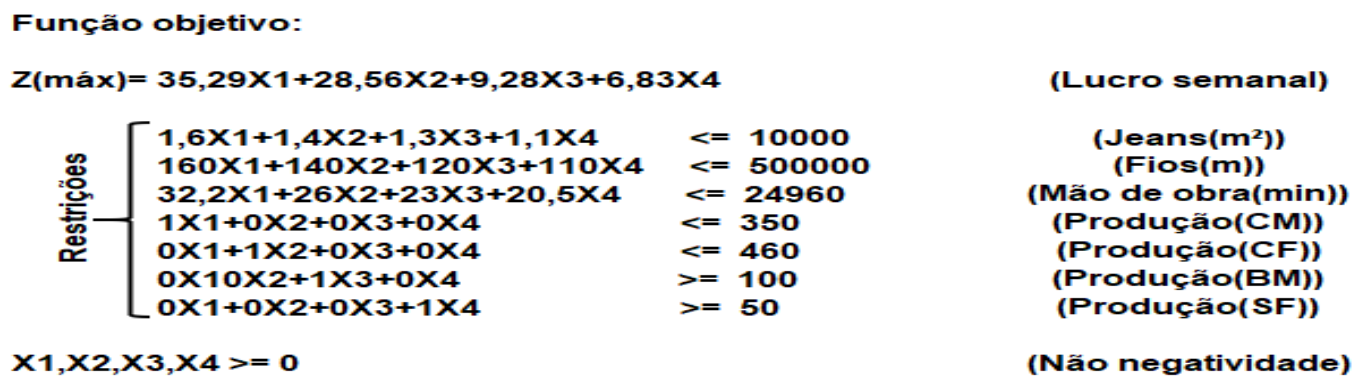

(Năo negatividade)

Figura 1- Modelagem matemática

A modelagem matemática mostra todos os recursos que são utilizados no processo de produção das peças de jeans e suas restrições, (limitações) da quantidade de fabricação dos produtos. Sabendo disso utilizou-se o software LINDO® para otimizar o processo, gerando informações com alta confiança e rapidez ajudando assim na tomada de decisão, que com essas restrições é possível fabricar determinadas quantidades de peças para ter o maior lucro semanal.

\section{Resultados}

A partir dessa ferramenta e com as informações dos dados, construíram-se fórmulas para moldar o problema, para que o mesmo possa ser resolvido com o programa LINDO®, que utiliza programação linear inteira, como mostra na Figura 2:

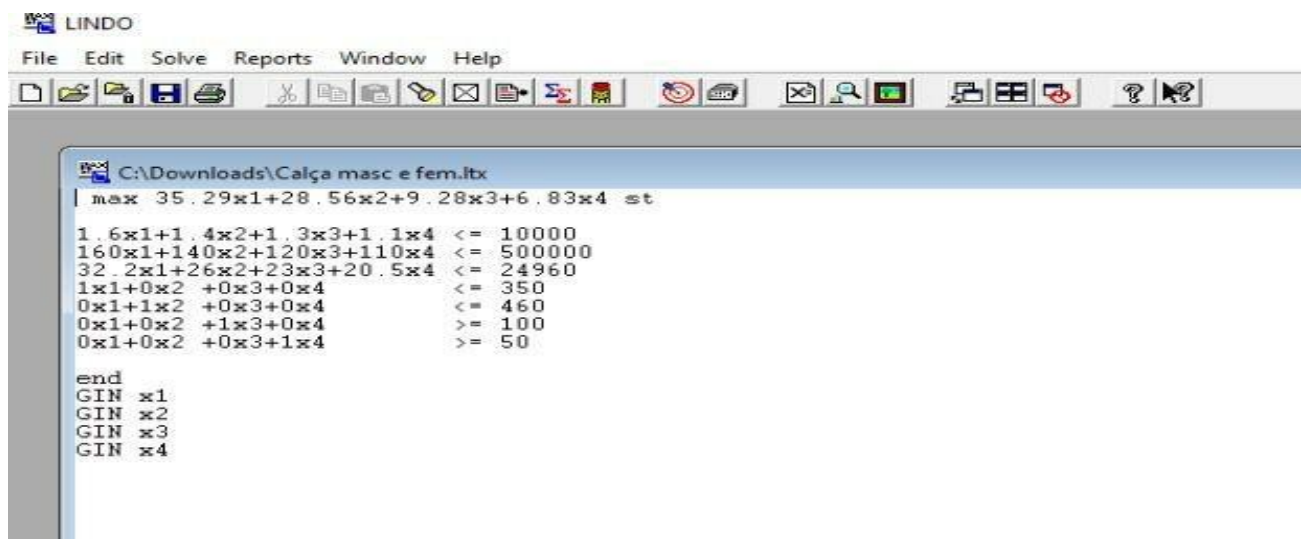

Figura 2- Modelagem matemática no LINDO 
Após a resolução obtida pelo LINDO® obedecendo todas as restrições, a quantidade que deverá ser produzida de cada modelo para se obter o maior lucro possível, e a quantidade gasta dos recursos para ter essa maximização, está sendo exposta na Tabela 3.

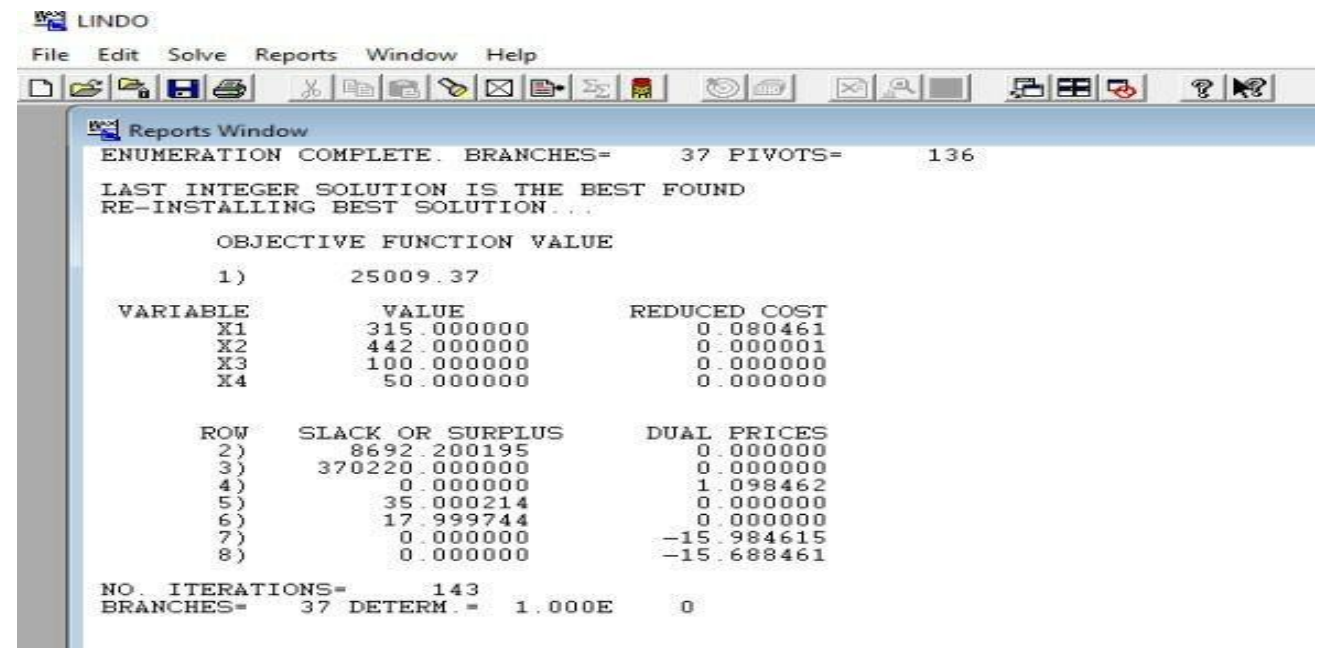

Figura 3 - Resultado matemática no LINDO

Tabela 6 - Quantidade que deverá ser produzida

\begin{tabular}{clccc}
\hline Calça masculina & Calça feminina & Bermuda masculina & Short feminino & LUCRO \\
\hline 315 & 442 & 100 & 50 & 25009,37 \\
\hline
\end{tabular}

Fonte: Elaborado pelos autores

É nítido observar que para ter o lucro máximo de $\mathrm{R} \$ 25009,37$ por semana, a empresa deveria fazer um total de 907 peças de jeans nesse período, na faixa de 151 peças por dia. Com esse resultado temos também as informações dos insumos que serão gastos e os insumos que sobraram na fabricação nesse período, como mostra na Tabela 7 e Tabela 8 respectivamente.

Tabela 7 - Quantidade de insumos que será gasto

\begin{tabular}{ccc}
\hline Jeans $\left(\mathbf{m}^{2}\right)$ & Fios $(\mathbf{m})$ & Mão de obra(min) \\
& & 24960 \\
\hline 1307,8 & 129780 & \\
\hline
\end{tabular}


Tabela 8 - Quantidade de insumos que sobraram

\begin{tabular}{ccc}
\hline $\operatorname{Jeans}\left(\mathbf{m}^{2}\right)$ & Fios(m) & Mão de obra(min) \\
\hline 8692,20 & 370220 & 0 \\
\hline
\end{tabular}

Fonte: Elaborado pelos autores

Observou-se que foi gasto toda Mão de obra disponível, mas sobrou uma quantia significativa de Jeans $\left(8692,20 \mathrm{~m}^{2}\right)$ e fios $(370220 \mathrm{~m})$, isso significa que está havendo muito desperdício de matéria prima.

\section{Conclusão}

Após a modelagem matemática e a obtenção dos resultados, foi possível constatar que o modelo proposto pode auxiliar de forma expressiva no planejamento da linha de produção têxtil, proporcionando agilidade, facilidade e confiabilidade nas análises realizadas, trazendo fácil entendimento do macroprocesso de produção propiciando assim uma melhor tomada de decisão para os gestores e melhoria dos resultados financeiros da organização. 


\section{Referências}

ARENALES, M. et. al. Pesquisa Operacional para cursos de Engenharia. Rio de Janeiro: Elsevier,2015.

COLIN, E. C.; CIPPARRONE, F. A. M.; SHIMIZU, T. Otimização do Custo de Transporte na

Distribuição Armazenagem de Açúcar. São Paulo: Escola Politécnica da USP, 1996.

BELFIORE, P. / FÁVERO, L.Pesquisa Operacional Para cursos de Engenharia-Rio de Janeiro-RJ:

Elsevier (2013)

COLIN, E. C.Pesquisa Operacional: 170 aplicações em estratégia, finanças, logística, produção, marketing e vendas-2. ed.- São Paulo: Atlas, 2018.

DANTAS, Rochelli. Pernambuco tem papel de destaque no setor têxtil. Disponível em:<https://www.diariodepernambuco.com.br/noticia/economia/2018/11/pernambuco-tem-papel-dedestaque-no-setor-textil.html> Acesso em 06 mar. 2020.

GODOY, A. S. Pesquisa qualitativa: tipos fundamentais. Revista de Administração de Empresas, São Paulo, 1995.

HILLER, F. S., LIBERMAN, G. J. Introdução a Pesquisa Operacional. 9a Ed. -Porto Alegre, RS: AMGH, 2013.

LACHTERMACHER, Gerson. Pesquisa Operacional na Tomada de Decisões, 5.ed. Rio de Janeiro: LTC, 2016.

MOREIRA, D. A. Pesquisa Operacional: Curso Introdutório. São Paulo: Thomson Learning, 2010.

SANTOS, Marcos dos; LIMA, I. C.; CARVALHO, F. B.; REIS, M. F.; SOUZA JUNIOR, Paulo Roberto. O uso da Programação Linear Inteira (PLI) no Apoio à Decisão e a Otimização do Mix de Produção. Anais do XXXVII Encontro Nacional de Engenharia de Produção. Joinville/SC, 2017.

SILVA, Ermes Medeiros da, SILVA, Elio Medeiros da, GONCALVES, Valter. Pesquisa operacional: programação linear, simulação. 3. ed. São Paulo: Atlas,2010.

ZANINE, Fábio. No agreste, polo de confecções serve de laboratório para reforma de Guedes. Disponível em:< https://www1.folha.uol.com.br/mercado/2019/04/no-agreste-polo-de-confeccoes-servede-laboratorio-para-reforma-de-guedes.shtml> Acesso em 06 de Mar. De 2020. 